\title{
ESTUDIO FITOQUÍMICO Y BIOQUÍMICO DE SEMILLAS PREHISPÁNICAS DE Nectandra sp.
}

\section{PHYTOCHEMICAL AND BIOCHEMICAL STUDY OF PREHISPANIC Nectandra sp. SEEDS}

\author{
Mag. María Montoya Vera ${ }^{a}$
}

\section{RESUMEN}

El presente articulo trata del estudio de un especímen botánico en particular, recurrentemente recuperado en diferentes sitios arqueológicos de la costa norte y central peruana. Este especímen ha sido identificado arqueobotánicamente como perteneciente a la familia Lauraceae, género Nectandra, especie aún no identificada, estas semillas son propias de zonas boscosas húmedas

La procedencia y ubicación cronológica del material arqueológico es el Complejo Arqueológico Huacas del Sol y la Luna ubicado en el valle bajo de Moche, material asociado a ocupación tardía Chimú (fechado C ${ }^{14} 1440$ - 1665 d. C.).

En el presente trabajo realizamos el análisis fitoquímico y los bioensayos con extractos de estas semillas, fueron realizados por especialistas en farmacología.

Actualmente estas semillas siguen siendo usadas en costa y sierra norte (herbolarios, curanderos), así como entre comunidades aguarunas de la selva norte.

Su significado simbólico está enmarcado en aspectos rituales, como un elemento ofrendatorio en complejas ceremonias funerarias, del cual hemos podido vislumbrar contextos recurrentes asociados a posibles sacrificios de niñosadolescentes en la época Chimú. Según los análisis realizados, las propiedades farmacológicas de estas semillas fueron conocidas y aprovechadas desde épocas preincaicas, tal y como las referencias etnohistóricas ya nos planteaban.

Palabras clave: Semillas, Nectandra sp., fitoquímico, bioquímico, rituales.

\section{ABSTRACT}

This article focuses on the study of a botanical specimen, that has been frcuently recovered in different archaeologic sites of the Peruvian coast north and central. Identified archaeobotanicly, it belongs to the Lauraceae family, gender Nectandra, kind yet unidentified, these seeds are own of forest areas wet

The origin and chronological location of the studied materials is Archaeologic Complex Huacas del Sol y la Luna located in the valley under of Moche, material associated with the late occupation Chimú (dated C ${ }^{14} 1440-1665$ a. C),

In the present work have accomplished the analysis phytochemical and bioensays with extracts of these seeds, they were accomplished by specialists in pharmacology

Currently its use continues being persistent, in coast and north saw (herbolarios, quacks), as well as between communities aguarunas of the north jungle.

Its meant symbolic is framed in ritual aspects, as an element to offer in complex funeral ceremonies, of which have been able to glimpse recurrent contexts associated with possible child sacrifices - adolescent for the era Chimú. Being apparently that the pharmacological properties of these seeds were known and taken advantage from the pre-Hispanic era, such and as the references ethnohistorics already were outlining us.

Key words: Seeds, Nectandra sp., phitochemical, biochemical, rituals.

\section{INTRODUCCIÓN}

La arqueología en las últimas décadas se ha visto enriquecida con la participación de diferentes disciplinas, reconociéndose así la verdadera importancia de rescatar de una manera más integral las evidencias dejadas por nuestras sociedades pasadas, permitiéndonos tener una visión más amplia para poder entenderlas mejor, brindándonos además nuevas posibilidades de investigación.

El presente trabajo tiene como lineamiento básico para su ejecución este principio, por lo cual para poder tener un conocimiento mas integral de un tipo específico de semillas que han venido siendo recuperadas de diferentes sitios arqueológicos de la costa norte y central peruana, y dada la escasa información sobre las mismas, nos vimos precisados a formular un estudio interdisciplinario, lo cual nos brindaría la oportunidad de entender mejor el rol que tenían en la época prehispánica.

Consideramos que las nuevas evidencias reportadas en el estudio fitoquímico de la Nectandra sp. son importantes, sin embargo reconocemos que aún faltan varios aspectos por dilucidar, y que esperamos entender mejor en el futuro. Esto nos refleja a su vez la necesidad de ejecutar más proyectos interdisciplinarios de análisis de especímenes arqueológicos, de los cuales aún sabemos muy poco, pero de los cuales se llega a especular muchas veces sin un sustento apropiado.

${ }^{a}$ Licenciada y Magister en Arqueología. Instituto Nacional de Cultura de Lambayeque. 


\section{MATERIAL Y METODOS}

\section{La Muestra}

Procede del sitio arqueológico Huaca de la Luna [1], ubicado en el valle bajo del río Moche, costa norte del Perú, consisten en semillas (Foto 01) recuperadas en una tumba Chimú fechada entre 1400-1515 d. C., fueron encontradas perforadas exprofesamente y traspasadas con soguillas, estaban dentro de paquetes textiles como ofrendas al personaje principal

enterrado $[3,4,5,6,7,8]$, se registró un total de 16,082 cotiledones (8,041 semillas). De acuerdo a la comparación inicial realizada en el Herbario de la UNT, y posteriormente en el Herbario del Museo de Historia Natural "Javier Prado" de la Universidad Nacional de San Marcos (Lima), se determinó que dichas semillas pertenecían al género Nectandra de la familia Lauraceae $[2,3]$.También se procedió a la identificación botánica de los especímenes comparativos modernos (Foto 01), los que han sido obtenidos en su mayoría en los puestos de venta de herbolarios y curanderos quienes denomina a estas mismas semillas como "Amala" (costa y sierra norte), "Matuc" o "Matuto" (selva norte), siendo muy raramente conocidos con el nombre de "Ishpingo" (Foto 01) $[2,3]$.

Las semillas arqueológicas así como también las modernas fueron sometidas a estudio fitoquímico, así como a una serie de bioensayos, para determinar propiedades específicas de los componentes identificados $[2,3]$, teniendo como objetivo principal contrastar su resultado con datos etnohistóricos.

\section{RESULTADOS}

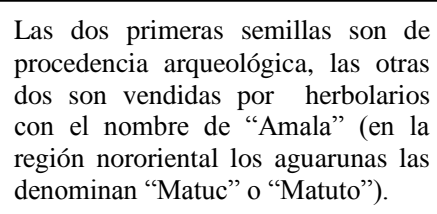

Las dos primeras semillas son de procedencia arqueológica, las otras dos son vendidas por herbolarios con el nombre de "Amala" (en la región nororiental los aguarunas las denominan "Matuc" o "Matuto")

Esta fila corresponde a semillas modernas que venden los herbolarios con el nombre de "Ishpingo".

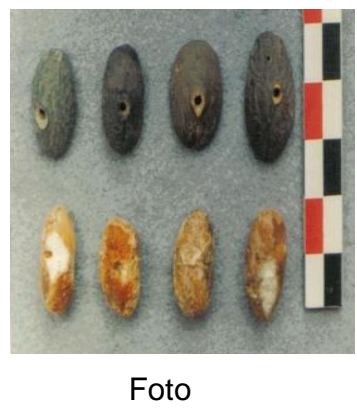

Foto

\section{Fotoquímico}

A. Estudio Fitoquímico de las muestras arqueológicas y modernas

\begin{tabular}{|c|c|c|c|c|}
\hline & Nombre científico & Nectandra sp. & Nectandra sp. & Amburana cearensis \\
\hline \multicolumn{2}{|c|}{ Componentes $\quad$ Nombre común } & $\begin{array}{c}\text { Semilla } \\
\text { arqueológica }\end{array}$ & $\begin{array}{l}\text { Semilla moderna } \\
\text { "Amala" }\end{array}$ & $\begin{array}{l}\text { Semilla moderna } \\
\text { "Ishpingo" }\end{array}$ \\
\hline \multirow[b]{2}{*}{ Alcaloides } & Prueba: Mayer & + & + & + \\
\hline & Prueba: Dragendorf & + & + & + \\
\hline \multicolumn{2}{|c|}{ Aminoácidos } & + & + & + \\
\hline \multicolumn{2}{|c|}{ Cardenolidos } & - & - & - \\
\hline \multicolumn{2}{|c|}{ Cumarinas } & + & + & + \\
\hline \multicolumn{2}{|l|}{ Esteroides } & - & - & - \\
\hline \multicolumn{2}{|c|}{ Flavonoides } & + & + & + \\
\hline \multicolumn{2}{|c|}{ Glucósidos cardiotónicos } & ++ & + & 0 \\
\hline \multicolumn{2}{|c|}{ Leucoantocianinas } & - & . & - \\
\hline \multicolumn{2}{|l|}{ Quinonas } & - & - & - \\
\hline \multicolumn{2}{|l|}{ Resinas } & + & + & + \\
\hline \multicolumn{2}{|l|}{ Taninos } & + & 0 & 0 \\
\hline
\end{tabular}

0 = Análisis no efectuado

\section{B. Bioensayos}

Bioensayo № 1: Administración de extracto alcohólico de las semillas de procedencia arqueológica (Nectandra sp.), para observar el efecto neuroestimulante, simulando la toma con chicha reportado en datos etnohistóricos.

Se realizó en un especímen del género Rattus rattus var. albinus, aparentemente sano, macho, peso aproximado de $250 \mathrm{mg}$.

I. Datos Basales: Las condiciones básales son de apariencia normal, excitable, despierta, responde a la llamada. Posteriormente se procedió a la administración del extracto vegetal, para la observación del cambio de estado de ánimo, así como de la actividad de la rata.

\section{Datos Pos-extracto vegetal:}

a. Primera dosis: $1 \mathrm{ml}$., vía intraperitoneal

\section{b. Controles pos-extracto vegetal:}

b.1 Diez minutos: S.N.C.: Efecto neuroestimulante, se pudo observar que la rata se encontraba excitada, los pelos posteriores erizados, estaba despierta, los ojos sobresalidos, bastante intranquila, con rápida respuesta al llamado, donde se pudo observar que con poca dosis de extracto el efecto es rápido.

b.2 Veinte minutos: S.N.C.: Efecto neuroestimulante, al observar luego de transcurrido un tiempo más prolongado se pudo apreciar que la rata se encontraba excitada, los pelos más erizados y con una marcada hiperreflexia.

\section{c. Segunda dosis: $1 \mathrm{ml}$. por vía intraperitoneal}

\section{d. Controles pos-administración de extracto vegetal:}

d.1 Diez minutos: S.N.C.: Efecto neuroestimulante, a la administración de una segunda dosis se pudo apreciar un cambio más notorio de la actividad de la rata, aún más excitada, despierta, los ojos sobresalidos, con marcada hiperreflexia, con lo que se comprobó su efecto neuroestimulante.

d.2 Veinte minutos: S.N.C.: Efecto neuroestimulante, luego de transcurrido más tiempo se pudo observar que la rata presentaba un pequeño malestar, debido al parecer a la liberación de histamina del organismo, así como de otros compuestos como la adrenalina, noradrenalina, etc., que ayudan a que se presenten estos efectos, los efectos principales que se pudo observar son el picadeo de la nariz, patas, genitales, etc. de la rata. Despierta, la hiperreflexia va disminuyendo, así como su actividad motora.

e. Tercera dosis: $1 \mathrm{ml}$. por vía intraperitoneal

\section{f. Controles pos-administración de extracto vegetal:}

f.1 Diez minutos: S.N. C.: Efecto neuroestimulante, después de la administración de una tercera dosis se pudo apreciar que la rata, fue perdiendo sus reflejos y la 
actividad motora, poco a poco, presentaba ataxia, e incoordinación, aún se mantenía despierta pero sin poder moverse, lo que demostró que el extracto tiene un efecto neuroestimulante, en una mayor dosis pudo apreciarse un efecto depresor.

\section{Bioensayo № 2: Reacción de alcaloides en cabello humano (Tumba 7) y pelo de la rata inyectada con extracto de la muestra arqueológica № 3 .}

El experimento llevado a cabo con la rata brindó la posibilidad de realizar un experimento comparativo entre el cabello humano de la tumba 7 de Huaca de la Luna y el pelo de la rata, el cual debía responder positivamente a la reacción de alcaloides, en la medida que los metabolitos se quedan impregnados en el pelo. Ambas muestras fueron colocadas en alcohol, realizándose pruebas cualitativas, tales como la reacción de Mayer, la cual dio positivo (cambio de color), y la reacción de Mandelin en que también cambió de color a rojo el precipitado, ambas pruebas fueron interpretadas como positivas y que por las características de la reacción el tipo de alcaloide podría ser del tipo Estricnina o Solanina. También fue realizada una tercera prueba, denominada la reacción de Ehrlich, en la que no se detectó un cambio a color violeta, determinándose que los alcaloides presentes no pertenecían al tipo Aminos.

Bioensayo № 3: Aplicación de extracto de semillas de Nectandra sp. (arqueológicas y modernas) y solución de estricnina en ratones, para comprobar mediante comparación la reacción a los alcaloides tipo estricnina reportadas en dichas semillas.

Los bioensayos fueron realizados en tres ratones (Mus musculus var. albinus) del Bioterio de la Facultad de Ciencias Biológicas, y el experimento fue desarrollado en los Laboratorios de Fitoquímica de la Facultad de Farmacia.

Bioensayo № 3a: Aplicación extracto semillas arqueológicas de Nectandra sp. El especímen estaba intranquilo al aplicarle la primera dosis $(0.1 \mathrm{~mL}$.), luego del cual no se observó mayor variación en su comportamiento. A los 30 minutos le fue aplicada una segunda dosis (similar), observándose cambios notables, primeramente picor en la nariz, luego se volvió tranquila, se apreció parálisis de los miembros inferiores, la respiración era rápida, no reaccionaba al llamado, empezando a dormitar, y también ataxia. Aproximadamente a los diez minutos de la segunda dosis el ratón empezó a convulsionar no muy fuerte, pero el ratón quedó muerto por parálisis muscular (tónico clónico)

- Bioensayo № 3b: Aplicación de extracto de semillas modernas de Nectandra sp. El ratón se encontraba intranquilo originalmente, al aplicársele la primera dosis (0.1 mL.) se volvió tranquilo y con picor en la nariz, se volvió lenta y torpe al caminar, apreciándose paulatinamente una parálisis de sus miembros inferiores, y ataxia. Se arrastra y estira, no reacciona al llamado y dormita un poco. A los 12 minutos de la primera dosis se apreciaron convulsiones con sacudidas leves, agitándose y respirando rápidamente, ésto se volvía cada vez más continuo, luego lentamente fue dormitando sin mayor reacción y con los ojos abiertos, moviéndose aproximadamente cada cinco minutos y volviendo a dormitar, produciéndose una parálisis muscular pero el especímen no murió.

- Bioensayo № 3c: Aplicación de estricnina (solución al 1 $x$ 1,000) El especímen en estado normal estaba inquieto, al aplicársele la solución sigue algo inquieto pero paulatinamente va tranquilizándose, movilizándose lentamente y produciéndose de pronto una fuerte convulsión (tónico clónica) cayendo muerta a los tres minutos de la aplicación de la única dosis.

Los resultados confirman que las semillas de Nectandra sp. contienen alcaloides del tipo estricnina (las pruebas sugieren una mayor concentración de alcaloides en las semillas de procedencia arqueológica que en las modernas), por lo tanto son tóxicas, produciéndose la muerte por parálisis muscular. La diferente reacción en la rata y en ratones nos haría pensar que la dosis letal depende de la contextura del especímen que ingiere en este caso el extracto de semillas de Nectandra.

El carácter alucinógeno de los alcaloides (presentes en estas semillas) no ha podido ser demostrado aún, los experimentos para demostrar ésto deberán hacerse con otro tipo de especímenes (gatos, etc.). Al respecto, es factible que estas semillas puedan tener efectos alucinógenos pero debemos investigar también qué otro tipo de alcaloides (aparte del tipo estricnina) contienen.

\section{Bioensayo № 4: Reacción anticoagulante}

Se utilizaron semillas arqueológicas y sangre humana.

\section{Procedimiento}

Para realizar este estudio se procedió a realizar una punción venosa en el brazo izquierdo para obtener la sangre a utilizar. En un tubo de ensayo conteniendo $2 \mathrm{ml}$. del extracto de las semilla 2 , y otro de la semilla 3 , se adicionó la sangre, para observar si presenta efecto anticoagulante.

\section{FUNDAMENTO}

Los anticoagulantes se emplean para prevenir o reducir las secuelas tromboembólicas del daño vascular atribuible a enfermedad cardiaca, cirugía, neoplasia o trauma. La coagulación de la sangre es vulnerable a la acción de los fármacos en diversos puntos del proceso.En el extracto de las semillas se encuentra una cantidad apreciable de Cumarinas, lo que nos llevó a ver si presentaba efecto anticoagulante, teniendo presente que el dicumarol es un fármaco que se usa para este fin y que químicamente es una Coumarina.

\section{CONCLUSIÓN}

El experimento reportó que el extracto de la semilla tiene efecto anticoagulante, pero de duración muy corta, lo que se puede explicar porque los anticoagulantes de Cumarinas son efectivos sólo in vivo, al interferir con la síntesis de los factores II, VII, IX y X dependientes de la vitámina $\mathrm{K}$, y por un bloqueo del paso de carboxilación requeridos para la producción de factores activos. 
Así mismo la gran variabilidad individual en la respuesta a los anticoagulantes de Coumarina se debe a muchos factores diferentes, incluyendo la velocidad de absorción y transformación metabólica, dieta y resistencia determinada genéticamente a estas sustancias.Por lo tanto se puede decir que este efecto se podría observar mejor al ser administrado a animales de experimentación, para poder observar mejor el efecto anticoagulante.

\section{DISCUSIÓN}

Si bien los experimentos fueron realizados solo en un especímen Rattus rattus var. albinus y en tres ratones de la especie Mus musculus var. albinus, lo cual constituye una limitación en la generalización de los resultados obtenidos, consideramos que éstos pueden ser tomados como indicativos de las reacciones reportadas, y que solo podrá verificarse cuando se realicen nuevos bioensayos controlando las reacciones en tres grupos de ratas (con tres animales por grupo) como mínimo. A pesar de esto, hemos realizado una correlación con los datos etnohistóricos (considerando que estas semillas son las denominadas "espingo" o "espinco" por los cronistas), y observamos que efectivamente se correlacionan con las reacciones evidenciadas en el estudio bioquímico.

La actividad analgésica se estaría relacionando a lo reportado por los cronistas[9], quien mencionaba sobre el "espinco": "Los polvos desta yerba, mezclados con polvos de incienso y dados en vino, hacen no sentir los tormentos por rigurosos que sean".

Mencionan en relación a la bebida de chicha mezclada con polvo de "espingo": "... beben la demás los hechiceros, y les vuelve como locos" [10], lo cual estaría relacionado a la actividad de los alcaloides de las semillas en el sistema nervioso.

La actividad anticoagulante, según los resultados obtenidos, nos dicen que, en sangre fresca el extracto de las semillas de Nectandra sp. tiene corta duración, siendo que al parecer ésto dependería de su condición "in vivo" de la sangre, por lo que la ingesta 0 el tratamiento con estos especímenes puede muy bien ser positivos para los casos mencionados por los cronistas, quienes citan sobre el "espingo": "... con sus polvos, tomados en ayunas, en agua o vino aguado, se curan las cámaras de sangre, y tomadas por el mismo orden o en alguno de los lamedores de rosas secas o de arrayán, son contra el flujo de sangre de vena rota en el pecho, y contra el que sale por la vía de la orina" [9], o cuando mencionan: "...; dicen que es muy medicinal para dolores de estómago, y cámaras de sangre y otras enfermedades tomado en polvos, ..." [10].

Así también existen referencias sobre el "espingo" y su característica peculiar de semejar posiblemente la sangre: "... cuya sustancia, cuajada, es como sangre de drago, aunque reluciente y tirante a negra, y de suave y profundo olor ..." [9].

Esta última referencia bien podría hacer pensar sobre la simulación artificial de la sangre, pudiéndose haber utilizado para fines ceremoniales en donde la sangre humana por su coagulación rápida les impediría mantenerla en forma líquida. Al respecto existen referencias sobre la actividad anticoagulante de otro especímen botánico, al parecer especie extinguida actualmente, ésta podría haber sido aprovechada en ciertas ceremonias en que hacían uso de sangre, tal y como se observa en algunas escenas Moche, esta propiedad generalmente ha sido atribuida al "ullucho", el cual ha sido estudiada en la iconografía Moche[11], e identificada [12] como un fruto de la familia Caricaceae, género y especie Carica candicans A. Gray. En los estudios fitoquímicos sobre el "ullucho" [13], se ha propuesto que el efecto anticoagulante en este especímen es provocado por la enzima papaína propia de las Carica, disolviéndose así la sangre coagulada por efecto de la proteína fibrina [13]. Si consideramos el reporte fitoquímico de la Nectandra sp. y su correlación con su identificación iconográfica podemos proponer que los Moche debieron conocer también las propiedades de las semillas de Nectandra sp., si bien no sabemos aún si fue usada en similar contexto ceremonial.

Sin embargo, en el conocido tema de la "Ceremonia de Sacrificio", así como en escenas de caza, etc. [3]

Se han podido observar elementos flotantes que se familiarizan con semillas de Nectandra sp., siendo probable que, así como se plantea que el "ullucho" fue usado en ceremonias donde la sangre era un elemento importante (sino el principal) en la que no debería coagularse durante un determinado periodo de tiempo, así también la Nectandra pudo usarse en este tipo de ceremonias.Debemos mencionar, que uno de los primeros sitios donde ha podido reportarse especímenes botánicos de "ulluchu" fue la tumba real del "Viejo Señor de Sipán", sin embargo la poca consistencia de los mismos no permitió una identificación botánica precisa (com. pers. W. Alva y F. León, 1996). Se considera, según la identificación realizada por el Dr. D. Ugent y F. León, que este especímen pertenecería a la familia de las meliáceas [14], considerándose las propiedades tóxicas y hasta letales (al parecer propias de esta familia) deberá replantearse las investigaciones anteriores sobre el "ulluchu" y considerar las implicancias de la nueva identificación para interpretar la iconografía Moche.

El consumo de bebidas conteniendo porcentajes de alcohol, de por si produce un efecto neuroestimulante, si consideramos el agregado a la chicha de especímenes que contienen alcaloides, como es el caso de la Nectandra sp. o "polvos de Espingo" citado por los cronistas, el efecto es mucho mayor, por lo que de manera aún preliminar (hasta que se realicen los análisis pendientes) proponemos el uso de este especímen como un aditivo que pudo haber provocado una muerte sin dolor, es decir la víctima pudo haber sido narcotizada, en ceremonias donde la ofrenda humana formaba parte del ritual a ejecutarse, precisamente habíamos ya propuesto $[3,7]$ que la presencia recurrente de niños-adolescentes sin causas especificas de muerte bien podrían explicarse a través del uso de estos especímenes botánicos, ésta propuesta no debe descartarse de plano y más bien valdría incidir sobre este aspecto en nuevas investigaciones interdisciplinarias.Según los contextos de aparición, tanto de la Nectandra como del "ulluchu", es indudable que ambos eran elementos aprovechables por la élite para determinados fines socio-políticos, enmarcados sutilmente en contextos ceremoniales. 
Futuros estudios deberán especificar mejor los componentes activos de estos especímenes botánicos y la explicación de su presencia en determinados contextos arqueológicos

\section{AGRADECIMIENTOS}

Un agradecimiento especial a los siguientes especialistas, quienes realizaron la certificación botánica,Dr. Manuel
Fernández Honores de la Facultad de Ciencias Biológicas de la UNT, y la certificación fitoquímica Q. F. Rosa Rea V. y Microb. Abraham Luyo P. de la Universidad Nacional de Trujillo.

\section{REFERENCIAS BIBLIOGRÁFICAS}

[1] Uceda, S.; R. Morales; J. Canziani; María Montoya. Investigaciones sobre la Arquitectura y relieves policromos en Huaca de la Luna, valle de Moche. MOCHE: PROPUESTAS Y PERSPECTIVAS (Edit. E. Mújica y S. Uceda). Actas del I Coloquio de Arqueología sobre la Cultura Moche. Serie Travaux de I'Institut Français d'Etudes Andines. 1994 (79): 251-303. IFEA, FOMCIENCIAS, UNT.

[2] Montoya Vera, María. Semillas rituales prehispánicas en Huaca de la Luna, Valle de Moche: Estudio interdisciplinario. Informe Final del Proyecto de Investigación financiado por el Concejo Nacional de Ciencia y Tecnología (CONCYTEC). Lima. 1998.

[3] Montoya Vera, María. Semillas rituales de Nectandra sp. en las culturas Moche y Chimú: Estudio interdisciplinario. Tesis para obtener el grado de Magíster en Ciencias Sociales con mención en Arqueología. Escuela de Postgrado. Universidad Nacional de Trujillo. 1998.

[4] Montoya Vera, María. Implicancias del Estudio de Semillas Rituales en la Época Prehispánica. Revista del Museo de Arqueología. 1998 (6): 203-219. Universidad Nacional de Trujillo.

[5] Montoya Vera, María. Complejo de Ofrendas Rituales en el Período Intermedio Tardío. Revista Arqueológica SIAN. 1998 (5): 9-12. Trujillo.

[6] Montoya Vera, María. Polvos de Espingo. Revista Arqueológica SIAN. 1999 (8): 5-17. Trujillo.

[7] Montoya Vera, María. Complejo de ofrendas rituales y su asociación a sacrificios humanos de niños en la época Chimú, Valle de Moche. Desarrollo Arqueológico de la Costa Norte del Perú. 2004 (Tomo 2): 27-48. L. Valle A. editor. Ediciones SIAN.

[8] Montoya Vera, María. Análisis Arqueofaunístico de las Tumbas 7 (Chimú) y 8 (Moche) de Huaca de la Luna. Valle de Moche. Revista del Museo de Arqueología, Antropología e Historia. Facultad de Ciencias Sociales de la Universidad Nacional de Trujillo. 2006: 229-253.

[9] Cobo, Bernabé. Historia del Nuevo Mundo. Biblioteca de Autores Españoles. Ediciones Atlas. Madrid. (1964 (1653))

[10] Arriaga, Pablo José de. Extirpación de la Idolatría del Perú. Biblioteca de Autores Españoles. Tomo CCIX. Edic. Atlas. Madrid. Crónicas Peruanas de Interés Indígena. (1968 (1621)).

[11] McClelland, Donna D. The Ulluchu: A Moche Symbolic Fruit. Pre-Columbian Art History. Selected Readings. Cordy-Collins y Stern (Eds.). 1977: 435-452. Peak Publication. Palo Alto.

[12] Wassén, Henry. El 'Ulluchu' en la Iconografía y Ceremonias de Sangre Moche: La Búsqueda de su Identificación. Boletín del Museo Chileno Arte Precolombino. 1989 (3): 25-45. Santiago de Chile.

[13] Hultin, E.; Wassén, S. H. y Bondenson, W. Papain in Moche Blood Ceremonies. Journal of Ethnopharmacology. 1987 (19): 227-228. Elsevier Scientific Publishers Ireland Ltd.

[14] Alva A., Walter. Sipán. Colección Cultura y Artes del Perú. Edición de José Antonio de Lavalle. Cervecería Backus y Johnston S.A. 1994.

E-mail: marianectandra@hotmail.com. 